\title{
The Coverage-Capacity Tradeoff in Cellular CDMA Systems
}

\author{
Venugopal V. Veeravalli, Senior Member, IEEE, and Andrew Sendonaris, Member, IEEE
}

\begin{abstract}
In cellular CDMA systems that employ single-user detectors, in-cell interference limits the coverage of the cell. Thus for a given upper limit on transmit power, the coverage of a cell is inversely proportional to the number of users in it. This tradeoff between coverage and number of users is explicitly characterized here. Our analysis may be used in cellular planning to set hard limits on the number of users admitted into the cell in order to meet coverage requirements. Furthermore, our approach allows us to arrive at a precise definition for the pole capacity of a cell, which serves as an upper bound on the number of users a cell can support as the coverage shrinks to zero. We also present a technique to calculate cell coverage as a function of carried traffic, for any given admission policy.
\end{abstract}

Index Terms - Capacity, CDMA, cellular systems, coverage, pole capacity.

\section{INTRODUCTION}

A $\mathrm{N}$ ACCURATE prediction of cell coverage as a function of user capacity is essential in code-division multiple access (CDMA) network design and deployment, and is therefore of great interest. Cell coverage is defined as the maximum distance that a given user of interest can be from the base station and still have a reliable received signal strength at the base station.

In cellular CDMA systems with nonorthogonal users and single-user detection (such as the reverse-link of IS-95 [1]) it is well known that the coverage of a cell has an inverse relationship with the user capacity of the cell. An increase in the number of active users in the cell causes the total interference seen at the receiver to increase. This causes an increase in the required received power for each user, due to the fact that each user has to maintain a certain signal-tointerference ratio at the receiver for satisfactory performance. For a maximum allowable transmit power, an increase in the required received power will result in a decrease in the maximum distance a mobile can be from the base station, thereby reducing coverage. We will assume that coverage is limited by the maximum transmit power at the mobile, although for some systems the forward link might be the limiting link [2].

Manuscript received June 11, 1997; revised May 6, 1998. This work was supported in part by Nortel's Global External Research Program.

V. V. Veeravalli is with the School of Electrical Engineering, Cornell University, Ithaca, NY 14853 USA (e-mail: venu@ee.cornell.edu).

A. Sendonaris was with the Department of Electrical and Computer Engineering, Rice University, Houston, TX 77005 USA. He is now with Qualcomm, Inc., Santa Clara, CA USA (e-mail: sendos@qualcomm.com).

Publisher Item Identifier S 0018-9545(99)07385-5.
Previous analyses of CDMA cell coverage have mainly focused on the extension of cell coverage that results from soft and hard handoff [3]-[6]. Our goal is to derive an analytical relationship between coverage and capacity. To facilitate the analysis, we do not include the effects of soft handoff and sectorization - the extension of our analysis to these cases is discussed in Section VII.

We first consider the case where the number of users in the cell is deterministic and the other-cell interference density is known. For this case, we derive an equation relating the coverage and number of users, for a given upper bound on outage probability. We also derive an equation for the pole capacity of a cell, i.e., the maximum number of users a cell can support if there is no constraint on the peak received power.

We then extend the analysis to the case where the number of users is random. Here we provide equations that can be used to obtain a tradeoff curve between cell coverage and the average number of users, for an arbitrary distribution on the number of users. This analysis will allow prediction of coverage for a projected capacity (carried traffic), irrespective of the admission policy ${ }^{1}$ used to achieve that capacity or the resulting call blocking probability.

The remainder of this paper is organized as follows: in Section II, we introduce the notation used in the paper and derive an expression for the outage probability. In Section III, we use the condition for power control feasibility to precisely define cell pole capacity. Then, in Section IV, we derive an explicit equation for coverage as a function of the number of users in the cell. This analysis is extended to the random user case in Section V, where a relationship between coverage and carried traffic is derived for a general admission policy. Section VI provides numerical results that illustrate our analysis techniques. Our conclusions are given in Section VII.

\section{Problem Formulation}

We will begin by introducing the relevant variables and the notation required for our analysis.

- For any power or signal-to-interference ratio variable $X$ expressed in decibels, $\hat{X}$ denotes $10^{X / 10}$.

- $k$ denotes the number of users in the cell, i.e., those being controlled by the cell's base station (BS).

\footnotetext{
${ }^{1}$ A "rise over thermal noise" admission policy [7] guarantees power control feasibility at the time of admission [8], and thus affects our probability of outage calculations. However, at all other times after admission, power control feasibility is not guaranteed, for this or any admission policy, and, therefore, needs to be considered during probability of outage calculations. We note that our calculations are independent of the admission policy.
} 
- $\hat{S}_{j}$ is the power received at the BS from the $j$ th user in watts. (The received power in decibel-watts is, by the above notation, given by $S_{j}=10 \log \hat{S}_{j}$.)

- $\hat{\varepsilon}_{j}$ is the signal-to-interference ratio (SIR or $E_{b} / I_{0}$ ) for the $j$ th user.

- $\nu_{j}$ is the voice activity factor of $j$ th user. The variables $\left\{\nu_{j}\right\}$ are modeled as independent Bernoulli random variables that take the value 1 with probability $\rho$, and the value 0 with probability $1-\rho$.

- $R$ denotes the information bit rate in bits per second.

- $W$ denotes the system bandwidth in hertz.

- $N_{0}$ is background noise power spectral density.

- $I$ is the other-cell interference density.

The signal-to-interference ratio (SIR) for the $j$ th user at the BS may be expressed in terms of the received powers of the various users as [7]

$$
\hat{\varepsilon}_{j}=\frac{\frac{\hat{S}_{j}}{R}}{\sum_{i: i \neq j} \frac{\nu_{i} \hat{S}_{i}}{W}+N_{0}+I} .
$$

The SIR requirements for the various users in the cell vary with time due to changes in the multipath fading environment and imperfections in power control. In particular, let $\hat{\varepsilon}_{j}^{\text {target }}$ denote the target SIR that is a function of the target frame error rate (FER) and the multipath conditions, and let $\delta_{j}^{\hat{\varepsilon}}$ denote the error in the power control algorithm. Then, the required SIR for the $j$ th user is given by

$$
\hat{\varepsilon}_{j}^{*}=\hat{\varepsilon}_{j}^{\text {target }} \delta_{j}^{\hat{\varepsilon}} .
$$

That is, $\hat{\varepsilon}_{j}^{*}$ is the SIR that the power control algorithm is demanding from the mobile at that particular point in time, even though the target SIR may be slightly different. Field trials reported in [9] have shown that the SIR requirements $\hat{\varepsilon}_{j}^{*}$ are well modeled by log-normal random variables. Furthermore, we can assume that the fading processes that cause the fluctuations in SIR requirements for the various users are independent. By the above discussion, we can model $\left\{\hat{\varepsilon}_{j}^{\star}\right\}$ at any given time by independent and identically distributed (i.i.d.) log-normal random variables.

In order to meet the SIR requirements $\left\{\hat{\varepsilon}_{j}^{\star}\right\}$, the required received powers $\left\{\hat{S}_{j}^{\star}\right\}$ must satisfy the power control equations

$$
\hat{\varepsilon}_{j}^{\star}=\frac{\frac{\hat{S}_{j}^{\star}}{R}}{\sum_{i: i \neq j} \frac{\nu_{i} \hat{S}_{i}^{\star}}{W}+N_{0}+I} .
$$

\section{A. Outage Equation}

If the SIR of a given user is lower than the desired value for a certain period of time, we have an outage, i.e., a noticeable degradation in call quality. If the outage lasts long enough, then the call is dropped. Ideally, we would like to limit the probability of outage (and, of course, call drop) to a small number. If estimates of the autocorrelation function of the fading experienced by the mobile are available, one can predict the outage probability along a particular given mobile trajectory [10]. But for our coverage analysis, we require a measure of service quality for the mobile user that encompasses all possible fading scenarios. As a result, a coverage analysis that depends on minimum duration outage [10] would be intractable, if not unrealistic. Our approach, therefore, is to simply look at the probability of instantaneous outage, that is, the event that the SIR falls below the required value at any time. The justification for this is two-fold.

First, this corresponds to a worst case outage probability measure. If we contain the probability of instantaneous outage to a small value, say $p_{m}$, then the probability of outage will necessarily be smaller than $p_{m}$. The reason is that any event corresponding to outage will always contain an instantaneous outage event. The precise relationship between outage and instantaneous outage depends on the nature of the fading experienced by the mobile. In the worst case, when the fading is perfectly correlated for a duration equal to the minimum duration for outage, the events will have the same probability. Thus containing the probability of instantaneous outage to $p_{m}$ guarantees that the worst case outage probability is also contained to $p_{m}$.

Second, it is clear that, due to the time correlation in the fading, whenever the SIR $\hat{\varepsilon}$ is below some threshold $\hat{\varepsilon}^{\star}$, even instantaneously, it will necessarily be below any other threshold $\hat{\varepsilon}^{\prime}>\hat{\varepsilon}^{\star}$ for a duration that is greater than or equal to the duration of being below $\hat{\varepsilon}^{\star}$. Therefore, we can address the minimum duration outage issue by analyzing instantaneous outage using an SIR threshold that is lower than the actual threshold. That is, given a fading environment, and given that the SIR should not fall below a threshold equal to $\hat{\varepsilon}^{\prime}$ for a period exceeding $\tau$, there exists a $\hat{\varepsilon}^{\star}<\hat{\varepsilon}^{\prime}$ such that the probability of instantaneous outage corresponding to $\hat{\varepsilon}^{\star}$ equals the probability of minimum duration outage corresponding to $\hat{\varepsilon}^{\prime}$ and $\tau$. Therefore, we are able to use a simpler analysis in order to determine coverage. In doing so, we not only avoid the complexity of the calculations involving minimum duration outage but, more importantly, we avoid the intractability of these calculations. The intractability is due to the fact that, for our coverage analysis, we need to take into account all possible fading scenarios that a mobile user may face in a given cellular environment. In practice, the desired $\hat{\varepsilon}^{\star}$ can be found empirically for different cellular environments, such as rural, urban, dense urban, highway, etc. Finding $\hat{\varepsilon}^{\star}$, however, is beyond the scope of our paper.

Based on the above discussion, we focus on the instantaneous outage event for user $j$, i.e., the event $\left\{\hat{\varepsilon}_{j}<\hat{\varepsilon}_{j}^{\star}\right\}$. There are two ways in which this event can happen: i) the power control equations of (3) do not have a feasible solution ${ }^{2}$ (call this event $A_{\text {out }}$ ) and ii) the power control equations have a feasible solution, but the maximum transmit power $S_{\max }$ at the mobile is exceeded (call this event $B_{\text {out }}$ ). Thus the probability of outage is given by

$$
P_{\text {out }}=P\left(A_{\text {out }}\right)+\left[1-P\left(A_{\text {out }}\right)\right] P\left(B_{\text {out }} \mid A_{\text {out }}^{c}\right)
$$

\footnotetext{
${ }^{2}$ That is, no matter how large the received powers are, the SIR requirements of the users cannot be satisfied.
} 
where $A_{\text {out }}^{c}$ is the complement of event $A_{\text {out }}$, i.e., $A_{\text {out }}^{c}$ is the event that the power control equations have a feasible solution.

We will use the outage equation to characterize the capacity-coverage tradeoff. To begin the analysis, we focus on the feasibility of the power control equations of (3).

\section{Power Control Feasibility and Pole Capacity}

It can be shown that the equations (3) have a feasible solution (i.e., $\left.\hat{S}_{j}^{\star} \in(0, \infty), \forall j\right)$ if and only if

$$
\sum_{i=1}^{k} \frac{R \hat{\varepsilon}_{i}^{\star} \nu_{i}}{W+R \hat{\varepsilon}_{i}^{\star} \nu_{i}}<1 .
$$

Under this condition, it can be shown that the required received power for user $j$ is given by

$$
\hat{S}_{j}^{\star}(k)=\frac{\frac{\left(N_{0}+I\right) W R \hat{\varepsilon}_{j}^{\star}}{W+R \hat{\varepsilon}_{j}^{\star} \nu_{j}}}{1-\sum_{i=1}^{k} \frac{R \hat{\varepsilon}_{i}^{\star} \nu_{i}}{W+R \hat{\varepsilon}_{i}^{\star} \nu_{i}}} .
$$

The above solution to the power control equations has been derived previously by other researchers [11]-[13]. For completeness, we have included a sketch of the derivation in the Appendix.

From (5), the probability that the power control equations of (3) do not have a feasible solution (event $A_{\text {out }}$ of (4)) is given by

$$
P\left(A_{\text {out }}\right)=P\left(\sum_{i=1}^{k} \frac{R \hat{\varepsilon}_{i}^{\star} \nu_{i}}{W+R \hat{\varepsilon}_{i}^{\star} \nu_{i}} \geq 1\right) .
$$

Under the log-normal assumption on required SIR's, the probability of event $A_{\text {out }}$ is always nonzero as long as $k \geq 2$, and $P\left(A_{\text {out }}\right)$ increases with increasing $k$. Thus even if there were no constraint on the maximum mobile transmit power, the probability that the SIR requirements are not met $\left(P_{\text {out }}\right)$ increases toward 1 with increasing $k$. This leads us to the following definition of pole capacity, that characterizes one extreme of the capacity-coverage tradeoff curve.

Definition 1: Let $p_{m}$ be the maximum allowable outage probability. The pole capacity $k_{\text {pole }}$ of a cell is the maximum number of users that can be accommodated in the cell such that $P_{\text {out }}<p_{m}$, if there is no constraint on the maximum received power for the various users.

From the above definition, it is clear that $k_{\text {pole }}$ serves as an upper bound on the maximum number of users that can be accommodated in the cell as the coverage of the cell shrinks to zero. This is due to the fact that for any finite maximum transmit power, the maximum received power increases dramatically as the coverage decreases toward zero. Numerical results given in Section VI will show that our coverage-capacity tradeoff curves do indeed approach $k_{\text {pole }}$ for shrinking coverage.

To calculate $k_{\text {pole }}$, we simply evaluate $P\left(A_{\text {out }}\right)$ as a function of $k$ and pick the largest value of $k$ such that $P\left(A_{\text {out }}\right)<$ $p_{m}$. For a given set of parameter values, $P\left(A_{\text {out }}\right)$ can be
TABLE I

$P\left(A_{\text {out }}\right)$ and Pole Capacity. The Parameter Values for This EXAmple ARE $W=1.25 \mathrm{MHz}, R=14.4 \mathrm{~kb} / \mathrm{s}$, $\rho=0.45, m_{\varepsilon}=7 \mathrm{~dB}$, AND $\sigma_{\varepsilon}=2.5 \mathrm{~dB}$. FOR A MAXIMUM Outage Probability $p_{m}=0.05$, We See That $k_{\text {pole }}=24$

\begin{tabular}{r|r|r}
\hline \multirow{2}{*}{$\mathrm{k}$} & \multicolumn{2}{|c}{$\mathrm{P}\left(A_{\text {out }}\right)$} \\
\cline { 2 - 3 } & Numerical convolution & Monte Carlo \\
\hline \hline 1 & 0.0000 & 0.0000 \\
\hline 2 & $2.4328 \mathrm{e}-13$ & \\
\hline 5 & $2.3803 \mathrm{e}-09$ & \\
\hline 8 & $4.5911 \mathrm{e}-07$ & - \\
\hline 11 & $1.8888 \mathrm{e}-05$ & $1.860 \mathrm{e}-5$ \\
\hline 14 & $2.9411 \mathrm{e}-04$ & $2.921 \mathrm{e}-4$ \\
\hline 17 & 0.0023 & 0.0023 \\
\hline 18 & 0.0041 & 0.0039 \\
\hline 19 & 0.0069 & 0.0073 \\
\hline 20 & 0.0112 & 0.0110 \\
\hline 21 & 0.0173 & 0.0175 \\
\hline 22 & 0.0257 & 0.0267 \\
\hline 23 & 0.0370 & 0.0368 \\
\hline 24 & 0.0516 & 0.0518 \\
\hline
\end{tabular}

computed for various $k$ using Monte Carlo techniques. Alternatively, we may obtain $P\left(A_{\text {out }}\right)$ as follows:

$$
\begin{aligned}
P\left(A_{\text {out }}\right) & =\sum_{\mathcal{A} \subset \mathcal{P}(\{1, \cdots, k\})} P(\mathcal{A}) P\left(\sum_{i \in \mathcal{A}} \frac{R \hat{\varepsilon}_{i}^{\star}}{W+R \hat{\varepsilon}_{i}^{\star}} \geq 1\right) \\
& =\sum_{j=0}^{k}\left(\begin{array}{c}
k \\
j
\end{array}\right) \rho^{j}(1-\rho)^{k-j} P\left(\sum_{i=1}^{j} \frac{1}{\alpha_{i}+1} \geq 1\right)
\end{aligned}
$$

where $\mathcal{A}$ denotes the set of all users for which $\nu_{j}=1$, $\mathcal{P}(\{1, \cdots, k\})$ denotes the set of all subsets of the set $\{1, \cdots, k\}$, and $\alpha_{i}=\left(W / R \hat{\varepsilon}_{i}^{*}\right)$. It can be shown that the pdf of $\left(1 /\left(\alpha_{i}+1\right)\right)$ is given by

$$
\begin{aligned}
f_{\left(1 /\left(\alpha_{i}+1\right)\right)}(x)= & \frac{10}{\ln 10} \frac{1}{\sqrt{2 \pi \sigma_{\varepsilon}^{2}}} \frac{1}{x(1-x)} \\
& \cdot \exp \left(-\frac{\left(10 \log _{10}\left(\frac{R}{W} \frac{1-x}{x}\right)+m_{\varepsilon}\right)^{2}}{2 \sigma_{\varepsilon}^{2}}\right)
\end{aligned}
$$

for $0<x<1$, where $m_{\varepsilon}$ and $\sigma_{\varepsilon}^{2}$ are the mean and variance, respectively, of $10 \log _{10} \hat{\varepsilon}_{i}^{\star}$. Due to the fact that the $\hat{\varepsilon}_{i}^{\star}$ are i.i.d., the probability density function (pdf) of $\sum_{i=1}^{j} \frac{1}{\alpha_{i}+1}$ may be obtained by repeated numerical convolution of the pdf given in (9) with itself. This leads to a numerical approximation of $P\left(\sum_{i=1}^{j} \frac{1}{\alpha_{i}+1} \geq 1\right)$ for any $j$, and thus to an approximation of $P\left(A_{\text {out }}\right)$. An example of this calculation is given in Table I, along with Monte Carlo simulation results.

As mentioned previously, $P_{\text {out }}$ depends not only on $P\left(A_{\text {out }}\right)$, but also on the probability that the required transmit power at the mobile exceeds the maximum allowed transmit power, i.e., on $P\left(B_{\text {out }}\right)$. As a result, as the number of users $k$ increases, the coverage of the cell decreases monotonically with essentially zero coverage in the vicinity of $k_{\text {pole. }}$. In the next section, we determine the coverage for all $k<k_{\text {pole }}$.

\section{Coverage Versus Number of Users}

Without loss of generality, we will focus on the coverage seen by user 1 when the number of users in the cell is $k$. Let 
$d$ be the distance of user 1 from BS. Then, the transmit power (in decibel-watts) of user 1 is given in terms of its received power $S_{1}$ at the BS by

$$
S_{\text {trans }}=S_{1}+\operatorname{PL}(d)+Z_{1}
$$

where $\mathrm{PL}(d)$ is the path loss at distance $d$ from the BS (including antenna gains) and $Z_{1}$ is a random variable representing shadow fading. The path loss is usually well modeled as (see, e.g., Hata's model [14])

$$
\operatorname{PL}(d)=K_{1}+K_{2} \log d .
$$

The shadow fading variable $Z_{1}$ is well modeled as a zero-mean Gaussian random variable with variance $\sigma_{Z}^{2}$ [14].

The probability of event $B_{\text {out }}$ for user 1 is the probability that $S_{\text {trans }}$ exceeds $S_{\max }$, the maximum power available at the mobile. Thus by (4), the probability of outage at a distance $d$ from the BS is given by

$$
\begin{aligned}
P_{\text {out }}= & P\left(A_{\text {out }}\right)+\left[1-P\left(A_{\text {out }}\right)\right] \\
& \cdot P\left(S_{1}^{\star}+\operatorname{PL}(d)+Z_{1}>S_{\max } \mid A_{\text {out }}^{c}\right) .
\end{aligned}
$$

The largest outage probability is seen at the edge of the cell. We can hence define the coverage of the cell $R_{\text {cell }}$ to be the distance from the BS at which $P_{\text {out }}$ equals the maximum allowable outage probability $p_{m}$. Thus $R_{\text {cell }}$ is obtained as a solution to

$$
\begin{aligned}
& P\left(A_{\text {out }}\right)+\left[1-P\left(A_{\text {out }}\right)\right] \\
& \quad \cdot P\left(S_{1}^{\star}+\operatorname{PL}\left(R_{\text {cell }}\right)+Z_{1}>S_{\text {max }} \mid A_{\text {out }}^{c}\right)=p_{m} .
\end{aligned}
$$

In (13), there are two quantities that depend on the number of users $k$ in the system: $P\left(A_{\text {out }}\right)$ and $S_{1}$. As demonstrated in Table I, $P\left(A_{\text {out }}\right)$ can easily be computed as a function of $k$-we denote this function by $P_{A}(k)$. Thus a relationship between coverage and number of users $k$ may be derived if we find the distribution of $S_{1}$, conditioned on $A_{\text {out }}^{c}$, as a function of $k$.

\section{A. Statistics of Received Power}

Conditioned on $A_{\text {out }}^{c}$, the power control equations have a feasible solution and this solution for user 1 is given by [see (6)]

$$
\hat{S}_{1}^{\star}(k)=\frac{\frac{\left(N_{0}+I\right) W R \hat{\varepsilon}_{1}^{\star}}{W+R \hat{\varepsilon}_{1}^{\star} \nu_{1}}}{1-\sum_{i=1}^{k} \frac{R \hat{\varepsilon}_{i}^{\star} \nu_{i}}{W+R \hat{\varepsilon}_{i}^{\star} \nu_{i}}} .
$$

This equation can be used in estimating the distribution of $\hat{S}_{1}^{\star}(k)$ via Monte Carlo techniques. In the following, we suggest a more useful way of approximating the pdf of $\hat{S}_{1}^{\star}(k)$.

The solution to the power control equations of (3) results in random variables $\hat{S}_{1}^{\star}, \hat{S}_{2}^{\star}, \cdots, \hat{S}_{k}^{\star}$ that are identically distributed; however, they are dependent in general. Nevertheless, numerical solutions to (3) for $k<k_{\text {pole }}$ show that variations in a particular $\hat{\varepsilon}_{j}^{\star}$ cause variations in $\hat{S}_{j}^{\star}$ that are at least an order of magnitude larger than variations in $\hat{S}_{i}^{\star}$, for any $i \neq j$ (see Table II). This suggests that $\left\{\hat{S}_{j}^{\star}\right\}$ are approximately
TABLE II

IndePendence of the Received Powers. This Table Demonstrates that the Percentage Change in Any Given $\hat{S}_{i}^{\star}, i \neq j$ Is Negligible Compared to the Percentage Change in $\hat{S}_{j}^{\star}$ Over a Wide Range of Variations of $\hat{\varepsilon}_{i}^{\star}$. In This Particular Example, $k=15, N_{0}=-169 \mathrm{dBm} / \mathrm{Hz}, I=2 N_{0}$, $W=1.25 \mathrm{MHz}, R=14.4 \mathrm{~kb} / \mathrm{s}, \rho=0.45, m_{\varepsilon}=7 \mathrm{~dB}$, AND $\sigma_{\varepsilon}=2.5 \mathrm{~dB}$

\begin{tabular}{r|r|l}
\hline \% change & \% change & $\max _{i \geq 2}\left\{\%\right.$ change in $\left.\hat{S}_{i}^{\star}\right\}$ \\
in $\hat{\varepsilon}_{1}^{\star}$ & in $\hat{S}_{1}^{\star}$ & \\
\hline \hline-20.0 & -20.2 & -0.6 \\
\hline-14.3 & -14.4 & -0.4 \\
\hline-8.6 & -8.7 & -0.3 \\
\hline-2.9 & -2.9 & -0.1 \\
\hline 2.9 & 2.9 & 0.1 \\
\hline 8.6 & 8.7 & 0.3 \\
\hline 14.3 & 14.5 & 0.4 \\
\hline 20.0 & 20.3 & 0.6 \\
\hline
\end{tabular}

independent for $k<k_{\text {pole }}$; we will use this approximation in our analysis.

From (3), the required SIR for the user 1 at the BS may be expressed in terms of the received powers of the other users as

$$
\hat{\varepsilon}_{1}^{\star}=\frac{\frac{\hat{S}_{1}^{\star}}{R}}{\sum_{i=2}^{k} \frac{\nu_{i} \hat{S}_{i}^{\star}}{W}+N_{0}+I} .
$$

Since the required SIR $\hat{\varepsilon}_{1}^{\star}$ is $\log$-normal, $\varepsilon_{1}^{\star}=10 \log \hat{\varepsilon}_{1}^{\star}$ is Gaussian. (Typical values for the mean and standard deviation of $\varepsilon_{1}^{\star}$ are $m_{\varepsilon}=7 \mathrm{~dB}$ and $\sigma_{\varepsilon}=2.5 \mathrm{~dB}$ [9].) If we let $m_{\hat{\varepsilon}}$ and $\delta_{\hat{\varepsilon}}$ denote the mean and second moment of $\hat{\varepsilon}_{1}^{\star}$, then we can easily show that

$$
m_{\hat{\varepsilon}}=\exp \left(\frac{\left(\beta \sigma_{\varepsilon}\right)^{2}}{2}\right) \exp \left(\beta m_{\varepsilon}\right)
$$

and

$$
\delta_{\hat{\varepsilon}}=\exp \left(2\left(\beta \sigma_{\varepsilon}\right)^{2}\right) \exp \left(2 \beta m_{\varepsilon}\right)
$$

where $\beta=\ln (10) / 10$.

Using the i.i.d. approximation for $\left\{\hat{S}_{1}^{\star}, \hat{S}_{2}^{\star}, \cdots, \hat{S}_{k}^{\star}\right\}$, we can obtain equations for all of the moments of $\hat{S}_{1}^{\star}$ by taking expectations of appropriate powers in (15). A moment analysis using four moments reveals that $\hat{S}_{1}^{\star}$ is very well approximated by a log-normal random variable for $k<k_{\text {pole }}$ (see Table III). Thus only the mean and second moment of $\hat{S}_{1}^{\star}$ need to be calculated. These are given by

$$
m_{\hat{S}}(k)=\frac{\left(N_{0}+I\right) W m_{\hat{\varepsilon}}}{\frac{W}{R}-\rho(k-1) m_{\hat{\varepsilon}}}
$$

and

$$
\delta_{\hat{S}}(k)=\frac{\left\{\left[\left(N_{0}+I\right) W+\rho(k-1) m_{\hat{S}}\right]^{2}-(k-1) \rho^{2} m_{\hat{S}}^{2}\right\} \delta_{\hat{\varepsilon}}}{\left(\frac{W}{R}\right)^{2}-\rho(k-1) \delta_{\hat{\varepsilon}}} .
$$

\footnotetext{
${ }^{3}$ A similar result could be obtained from (14) by approximating sums of log-normal random variables by log-normal rv's [15]. However, the presence of the Bernoulli voice activity factors $\nu_{i}$ could pose some additional problems.
} 
TABLE III

Log-Normal Approximation for Received Power. The Third Column Tabulates the First Four Moments of $\hat{S}_{1}^{\star}$, Derived Using (15), Whereas the Second Column Tabulates the Moments of a Log-Normal rv, That

Is, $E\left[X^{p}\right]=\delta_{X}^{p(p-1) / 2} / m_{X}^{p(p-2)}$ FOR $p>2$. IN THIS PARTICULAR

EXAMPLE, $k=15, N_{0}=-169 \mathrm{dBm} / \mathrm{Hz}, I=2 N_{0}, W=1.25$

$\mathrm{MHz}, R=14.4 \mathrm{~kb} / \mathrm{s}, \rho=0.45, m_{\varepsilon}=7 \mathrm{~dB}$, AND $\sigma_{\varepsilon}=2.5 \mathrm{~dB}$

\begin{tabular}{r|r|r}
\hline & If $\hat{S}_{1}^{\star}$ were $\log$-normal & Actual moments \\
\hline \hline $\mathrm{E}\left[\hat{S}_{1}^{\star}\right]$ & $5.63667 \mathrm{e}-15$ & $5.63667 \mathrm{e}-15$ \\
\hline $\mathrm{E}\left[\left(\hat{S}_{1}^{\star}\right)^{2}\right]$ & $4.55269 \mathrm{e}-29$ & $4.55269 \mathrm{e}-29$ \\
\hline $\mathrm{E}\left[\left(\hat{S}_{1}^{\star}\right)^{3}\right]$ & $5.26908 \mathrm{c}-43$ & $5.27060 \mathrm{c}-43$ \\
\hline $\mathrm{E}\left[\left(\hat{S}_{1}^{\star}\right)^{4}\right]$ & $8.73826 \mathrm{e}-57$ & $8.74817 \mathrm{e}-57$ \\
\hline
\end{tabular}
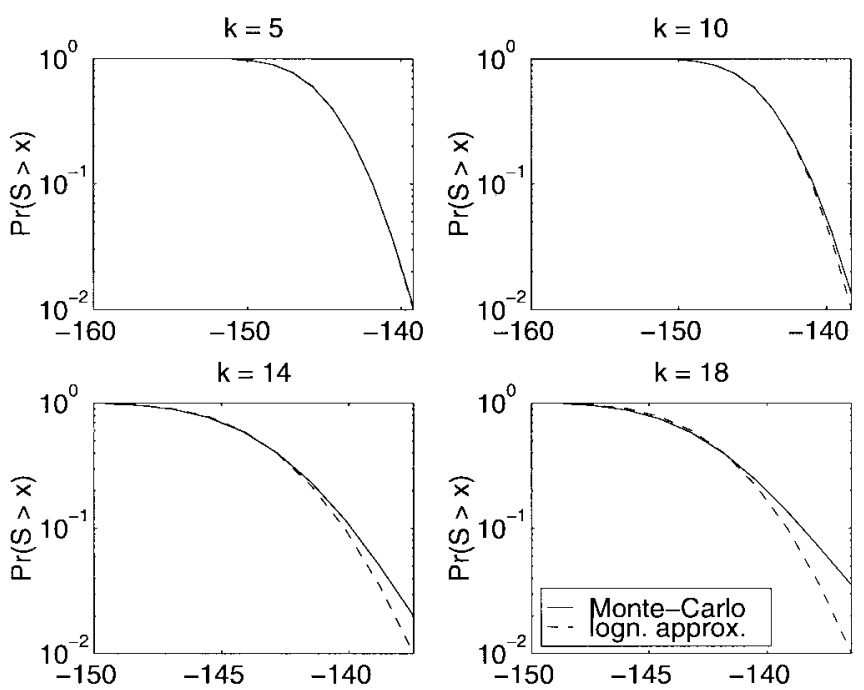

Fig. 1. Justification for independence assumption and log-normal approximation. The complementary cdf of $S_{1}^{\star}$ under the log-normal approximation is compared with that obtained via Monte Carlo techniques. The accuracy of the approximation decreases as $k$ approaches $k_{\text {pole }}$.

Under the log-normal approximation for $\hat{S}_{1}^{\star}, S_{1}^{\star}$ is Gaussian. The mean and variance of $S_{1}^{\star}$ can easily be calculated in terms of $m_{\hat{S}}$ and $\delta_{\hat{S}}$ as given below

$$
m_{S}(k)=20 \log _{10} m_{\hat{S}}(k)-5 \log _{10} \delta_{\hat{S}}(k)
$$

and

$$
\sigma_{S}^{2}(k)=\frac{1}{\beta}\left(10 \log _{10} \delta_{\hat{S}}(k)-20 \log _{10} m_{\hat{S}}\right) .
$$

As final justification for the independence assumption and the log-normal approximation for the $\hat{S}_{j}^{\star}$, we compare the complementary cumulative distribution function (cdf) of the approximation for $S_{1}^{\star}$ with the actual complementary cdf obtained from (14) using Monte Carlo techniques in Fig. 1. It is clear that the approximation is very good for $k \ll k_{\text {pole }}$. As $k$ approaches $k_{\text {pole }}$, the approximation is stochastically smaller than the actual required received power, indicating that the coverage estimate based on the log-normal approximation is slightly optimistic.

\section{B. Equation Relating Coverage and Number of Users}

Now, in order to evaluate the probability on the left-hand sider (LHS) of (13), we need to determine the joint statistics of $S_{1}^{\star}$ and $Z_{1}$. The means and variances of these random variables have been specified, so all that remains to be determined is the correlation between them. We can argue that the correlation between $S_{1}^{\star}$ and $Z_{1}$ is close to zero, since the fluctuations in the required received power $S_{1}^{\star}$ are mainly due to multipath fading and imperfections in power control, whereas the fluctuations in $Z_{1}$ are due to shadow fading. We can hence compute the conditional probability $P\left(B_{\text {out }} \mid A_{\text {out }}^{c}\right)$ in (13) as

$$
\begin{aligned}
& P\left(S_{1}^{\star}+\operatorname{PL}\left(R_{\text {cell }}\right)+Z_{1}>S_{\max } \mid A_{\text {out }}^{c}\right) \\
& \quad=Q\left(\frac{S_{\max }-\left(K_{1}+K_{2} \log R_{\text {cell }}\right)-m_{S}(k)}{\sqrt{\sigma_{S}^{2}(k)+\sigma_{Z}^{2}}}\right)
\end{aligned}
$$

where we have used the path loss model of (11), and where $Q(\cdot)$ is the complementary cdf of a zero-mean, unit-variance Gaussian random variable. Substituting (22) in (13), we get the following explicit equation relating the coverage $R_{\text {cell }}$ and the number of users $k$ :

$$
\begin{aligned}
\log R_{\text {cell }}=\frac{1}{K_{2}}[ & S_{\max }-K_{1}-m_{S}(k) \\
& \left.-\sqrt{\sigma_{S}^{2}(k)+\sigma_{Z}^{2}} Q^{-1}\left(\frac{p_{m}-P_{A}(k)}{1-P_{A}(k)}\right)\right]
\end{aligned}
$$

where $P_{A}(k)$ is $P\left(A_{\text {out }}\right)$ written explicitly as a function of $k$.

Numerical examples that describe $R_{\text {cell }}$ as a function of $k$ are given in Section VI.

Remark 1: It should be noted that as $R_{\text {cell }}$ approaches 0 in (23), $k$ approaches $k_{\text {pole }}$. However, the since the path-loss model we assumed is not valid for $d$ in the neighborhood of 0 , the actual limiting value of $k$ is strictly less than $k_{\text {pole }}$.

\section{Coverage Versus CAPACITY}

The equation for $R_{\text {cell }}$ as a function of $k$ derived in the previous section may be used in CDMA cellular network planning to set hard limits on the maximum number of users that can be admitted into the cell, such that prespecified coverage requirements are met. It may also be of interest in cellular planning to design cell coverages and capacities to match projected traffic densities in the network. In this case it may be appropriate to model the number of users in a cell as a random variable (denoted by $\kappa$ ). The statistics of $\kappa$ will be a function of the cell admission policy and the offered traffic. Our goal here is to derive a relationship between the mean of $\kappa$, which we denote by $c$, and cell coverage $R_{\text {cell }}$. We will refer to $c$, which represents the carried traffic in the cell, as capacity. Clearly, $c$ can be related to the Erlang capacity of the cell through the blocking probability [9].

Let $p_{\kappa}(\cdot)$ denote the probability mass function (pmf) of $\kappa$. Clearly, any useful admission policy will not allow $\kappa$ to exceed $k_{\text {pole, }}$ since power control becomes infeasible with probability greater than $p_{m}$ when the number of users exceeds $k_{\text {pole }}$. Power control feasibility can sometimes automatically be guaranteed by the admission policy. For example, in the admission policy suggested by Viterbi [7], users are admitted into the cell until the total interference seen at the base station exceeds the background noise level $N_{0}$ by a factor $\tau>1$. It is shown in [8] that this admission policy guarantees power control feasibility at the time of admission, which implies that 
the number of users admitted in the cell is smaller than $k_{\text {pole }}$ with high probability. However, to guarantee system stability at all times after admission, it would be necessary to always hard-limit the number of users to a maximum of $k_{\text {pole }}$. It might actually be preferable to hard-limit the users to a number that is considerably smaller than $k_{\text {pole }}$ to guarantee a minimum coverage.

In the following, we assume that support of $p_{\kappa}$ is limited to the set $\left\{0,1, \cdots, k_{\text {pole }}\right\}$. Conditioned on $\kappa=k, S$ is Gaussian with mean $m_{S}(k)$ and variance $\sigma_{S}^{2}(k)$. Thus we can compute the average outage probability at distance $d$ by averaging (12) over the distribution of $\kappa$ to get

$$
\begin{aligned}
\bar{P}_{\text {out }}(d)= & \frac{1}{1-p_{\kappa}(0)} \sum_{k=1}^{k_{\text {polc }}} p_{\kappa}(k)\left\{P_{A}(k)\right. \\
& \left.+\left[1-P_{A}(k)\right] P\left(S_{1}^{\star}+\operatorname{PL}(d)+Z_{j}>S_{\max } \mid A_{\text {out }}^{c}\right)\right\} .
\end{aligned}
$$

Hence the cell coverage $R_{\text {cell }}$ satisfies the equation

$$
\bar{P}_{\text {out }}\left(R_{\text {cell }}\right)=p_{m} .
$$

Given a model for the pmf of the number of users, we can use (25) to obtain a plot of coverage versus capacity. As an example, suppose $\kappa$ has a Poisson distribution ${ }^{4}$ with parameter $\xi$, truncated at $k_{\text {pole }}$, i.e.,

$$
p_{\kappa}(k)=\frac{\xi^{k} e^{-\xi} / k !}{\sum_{j=0}^{k_{\text {polc }}} \xi^{j} e^{-\xi} / j !}, \quad k=0,1, \cdots, k_{\text {pole }} .
$$

Then the average number of users in the cell is given by

$$
c=\xi\left[1-\frac{\xi^{k_{\text {polc }}} e^{-\xi} / k_{\text {pole }} !}{\sum_{j=0}^{k_{\text {polc }}} \xi^{j} e^{-\xi} / j !}\right] .
$$

By solving (25) and (27) for various values of $\xi$, we can obtain a tradeoff curve for $R_{\text {cell }}$ versus $c$ that is parameterized by $\xi$. An example of this calculation is given in Section VI.

\section{NUMERICAL RESULTS}

The parameter values used in our numerical results are as follows:

$$
\begin{array}{ll}
W=1.25 \mathrm{MHz} & \text { bandwidth; } \\
R=14.4 \mathrm{~kb} / \mathrm{s} & \text { bit rate; } \\
\rho=0.45 & \text { voice activity factor; } \\
m_{\epsilon}=7 \mathrm{~dB} & \\
\quad \text { (varied in Fig. 3) } & \text { median SIR required; } \\
\sigma_{\epsilon}=2.5 \mathrm{~dB} & \text { standard deviation of SIR required; } \\
K_{1}=17.3 \mathrm{~dB} & \text { path-loss constant; }
\end{array}
$$

\footnotetext{
${ }^{4}$ For an admission policy based on interference levels, the pmf is well modeled as Poisson [9].
}

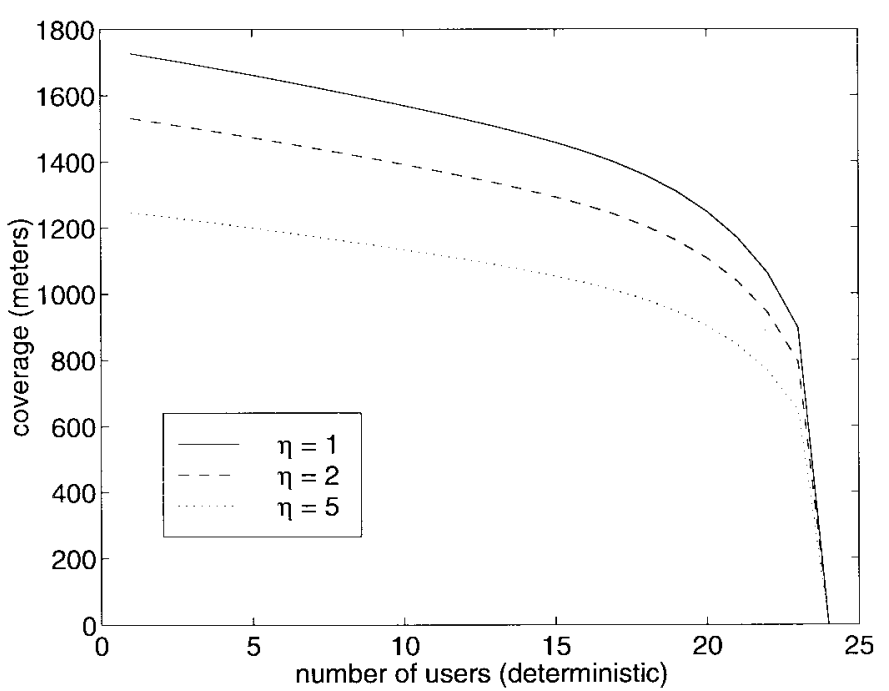

Fig. 2. Coverage versus number of users: sensitivity to variations in other-cell interference. The parameter $\eta$ denotes the ratio $I / N_{0}$.

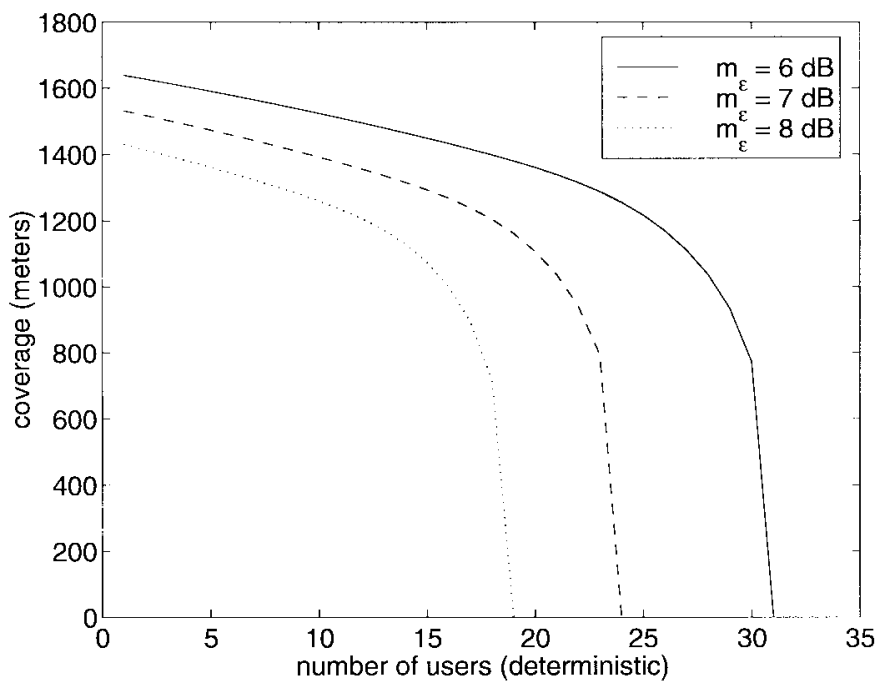

Fig. 3. Coverage versus number of users: sensitivity to variations in $m_{\varepsilon}$.

$$
\begin{array}{ll}
K_{2}=33.8 \mathrm{~dB} & \text { path-loss exponent; } \\
S_{\max }=23 \mathrm{dBm} & \text { maximum mobile transmit power; } \\
N_{0}=-169 \mathrm{dBm} / \mathrm{Hz} & \text { thermal noise PSD; } \\
p_{m}=0.05 & \text { maximum outage probability; } \\
\sigma_{Z}=8 \mathrm{~dB} & \text { shadow fading standard deviation }
\end{array}
$$

where $K_{1}$ and $K_{2}$ are obtained using Hata's model [14, p. 119] for a medium-sized city with carrier frequency of $900 \mathrm{MHz}$, transmit antenna height of $50 \mathrm{~m}$, receive antenna height of 1 $\mathrm{m}$, and a net antenna gain of $6 \mathrm{~dB}$.

Without loss of generality, we will assume that the othercell interference density $I$ is a multiple $\eta$ of the background thermal noise density $N_{0}$. In Fig. 2, we plot coverage versus number of users for various values of $\eta$, using (23). Note that the pole capacity is unaffected by $\eta$. In Fig. 3, we fix $\eta=2$ and vary the median SIR requirement $m_{\varepsilon}$. As expected, the tradeoff curves are considerably more sensitive to variations in $m_{\varepsilon}$ when the number of users is large. Finally, in Fig. 4, we 


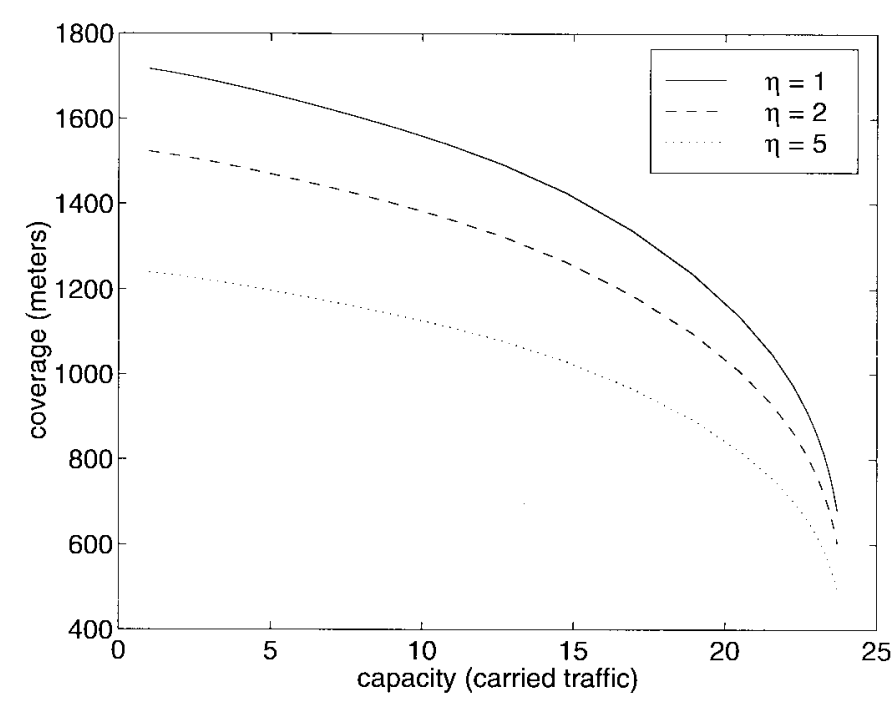

Fig. 4. Coverage versus capacity for a truncated Poisson user distribution. The parameter $\eta$ denotes the ratio $I / N_{0}$.

plot coverage versus capacity (carried traffic), using the model for $p_{\kappa}$ given in (26). It can be seen that the curves in Fig. 4 are lower than the corresponding curves in Fig. 2. This is to be expected due to the concavity of the capacity as a function of the number of users.

\section{CONCLUSIONS}

We derived an explicit relationship for the coverage of a cell as a function of the number of users in it. This relationship may be used in cellular planning for setting hard limits on the number of users admitted into a cell in order to meet coverage requirements. Our analysis also allowed us to arrive at a precise definition for the pole capacity of a cell.

We have also presented a way to determine the tradeoff between coverage and average number of users in the random user case. This technique may be used to characterize the capacity-coverage tradeoff for an arbitrary admission policy. Such a characterization would be particularly useful in optimally locating base stations in a geographical area based on prior information about traffic nodes.

Avenues for further research include extending the capacity-coverage analysis to incorporate the effects of using soft handoff, and of using sectorized cells. Extension to sectorized cells should be straightforward, with the understanding that the sectors in a given cell could, in general, have different capacity-coverage operating points. Incorporating soft handoff in the analysis is more complex. The outage condition would correspond to all of the base stations in soft handoff not meeting SIR requirements (assuming that selection diversity is used). Also, the analysis would need to incorporate the correlations between the shadow fading processes seen by the various base stations, and the correlations between their SIR requirements. Such an analysis would lead to a precise characterization of the improvement in the capacity-coverage tradeoff that results from soft handoff.

\section{APPENDIX \\ Solution to the Power Control Equations}

The solution to (3) is obtained as follows. We begin by defining the vectors

$$
\underline{\hat{S}}^{\star}=\left(\begin{array}{c}
\hat{S}_{1}^{\star} \\
\vdots \\
\hat{S}_{k}^{\star}
\end{array}\right) \quad \underline{\nu}=\left(\begin{array}{c}
\nu_{1} \\
\vdots \\
\nu_{k}
\end{array}\right) \quad \underline{\hat{\varepsilon}}^{\star}=\left(\begin{array}{c}
\hat{\varepsilon}_{1}^{\star} \\
\vdots \\
\hat{\varepsilon}_{k}^{\star}
\end{array}\right) .
$$

The equations in (3) may be rewritten in terms of these vectors as

$$
\begin{aligned}
\left(W \boldsymbol{I}+R \operatorname{diag}\left(\hat{\varepsilon}_{1}^{\star} \nu_{1}, \cdots, \hat{\varepsilon}_{k}^{\star} \nu_{k}\right)-R \hat{\underline{\hat{\hat{k}}}}^{\star} \underline{\underline{\nu}}^{T}\right) \underline{\hat{S}}^{\star} & =\left(N_{0}+I\right) W R \underline{\hat{\hat{\varepsilon}}}^{\star}
\end{aligned}
$$

where $I$ is the $k$-dimensional identity matrix. The matrix multiplying $\underline{\hat{S}}^{\star}$ in (29) is a rank-1 modification of the diagonal matrix $B$ given by

$$
\boldsymbol{B}=\boldsymbol{W I}+R \operatorname{diag}\left(\hat{\varepsilon}_{1}^{\star} \nu_{1}, \cdots, \hat{\varepsilon}_{k}^{\star} \nu_{k}\right) .
$$

It is easy to show that

$$
\left(\boldsymbol{B}-R \underline{\hat{\hat{\hat{k}}}}^{\star} \underline{\underline{\nu}}^{T}\right)^{-1}=\boldsymbol{B}^{-1}+\frac{R \boldsymbol{B}^{-1} \underline{\underline{\underline{\hat{\epsilon}}}}^{\star} \underline{\nu}^{T} \boldsymbol{B}^{-1}}{1-R \underline{\underline{\nu}}^{T} \boldsymbol{B}^{-1} \underline{\underline{\hat{\epsilon}}}^{\star}} .
$$

Thus the solution to (29) may be written as

$$
\begin{aligned}
\underline{\hat{S}}^{\star}= & \left(N_{0}+I\right) W R \boldsymbol{B}^{-1} \underline{\hat{\varepsilon}}^{\star} \\
& +\frac{\left(N_{0}+I\right) W R^{2} \boldsymbol{B}^{-1} \hat{\underline{\hat{\varepsilon}}}^{\star} \underline{\underline{L}}^{T} \boldsymbol{B}^{-1} \underline{\hat{\underline{\varepsilon}}}^{\star}}{1-R \underline{\boldsymbol{p}}^{T} \boldsymbol{B}^{-1} \underline{\hat{\underline{\varepsilon}}}^{\star}}
\end{aligned}
$$

which can be simplified to yield

$$
\underline{\hat{S}}^{\star}=\frac{\left(N_{0}+I\right) W \underline{V}}{1-\underline{\nu}^{T} \underline{V}}
$$

where

$$
V_{i}=\frac{R \hat{\varepsilon}_{i}^{\star}}{W+R \hat{\varepsilon}_{i}^{\star} \nu_{i}}, \quad i=1, \cdots, k
$$

It is easy to see that (33) is identical to (6). It is also clear that the solution given in (33) is feasible if and only if

$$
\underline{\nu}^{T} \underline{V}<1
$$

which is the condition given in (5).

\section{ACKNOWLEDGMENT}

The authors wish to thank N. Jain for his contributions to this paper. They also wish to thank S. Madhavapeddy and K. Basu for helpful discussions.

\section{REFERENCES}

[1] TIA/EIA/IS-95, Mobile Station-Base Station Compatibility Standard for Dual-Mode Wideband Spread Spectrum Cellular Systems, Telecommun. Industry Assoc., July 1993. 
[2] C. Wheatley, "Trading coverage for capacity in cellular systems: A systems perspective," Microwave J., vol. 38, no. 7, pp. 62-76, July 1995.

[3] A. J. Viterbi et al., "Soft handoff extends CDMA cell coverage and increases reverse link capacity," IEEE J. Select. Areas Commun., vol. 12, pp. 1281-1288, Oct. 1994

[4] Y. W. Chang and E. Geraniotis, "Accurate computations of cell coverage areas for CDMA hard and soft handoffs," in Proc. 1996 IEEE Vehicular Technology Conf., Atlanta, GA, May 1996, pp. 411-415.

[5] P. Patel et al., "A simple analysis of CDMA soft handoff gain and its effect on the cell's coverage area," Wireless Information Networks, J. Holtzman, Ed. Boston, MA: Kluwer, 1996, pp. 155-172.

[6] M. Chopra, K. Rohani, and J. D. Reed, "Analysis of CDMA range extensions due to soft handoff," in Proc. 1995 IEEE Vehicular Technology Conf., Chicago, IL, July 1995, pp. 917-921.

[7] A. J. Viterbi, CDMA: Principles of Spread Spectrum Communication. Reading, MA: Addison-Wesley, 1995.

[8] A. Sampath, N. B. Mandayam, and J. M. Holtzman, "Erlang capacity of a power controlled integrated voice and data CDMA system," in Proc. 1997 IEEE Vehicular Technology Conf., Phoenix, AZ, May 1997, pp. $1557-1561$

[9] A. M. Viterbi and A. J. Viterbi, "Erlang capacity of a power controlled CDMA system," IEEE J. Select. Areas Commun., vol. 11, pp. 892-899, Aug. 1993.

[10] N. B. Mandayam, P.-C. Chen, and J. M. Holtzman, "Minimum duration outage for cellular systems: A level crossing analysis," in Proc. 1996 IEEE Vehicular Technology Conf., Atlanta, GA, May 1996, pp. 879-883.

[11] A. Sampath, P. S. Kumar, and J. M. Holtzman, "Power control and resource management for a multimedia wireless CDMA system," in Proc. 1995 Conf. Personal, Indoor and Mobile Radio Communication, Toronto, Ont., Canada, Sept. 1995, pp. 21-25.

[12] S. V. Hanly, "Information capacity of radio networks," Ph.D. dissertation, Univ. Cambridge, Cambridge, U.K., 1993.

[13] L. Yun and D. Messerschmitt, "Variable quality of service in CDMA systems by statistical power control," in Proc. 1994 IEEE Int. Conf. Communications, Seattle, WA, June 1995.

[14] T. S. Rappaport, Wireless Communications: Principles and Practice. Upper Saddle River, NJ: Prentice-Hall, 1996.

[15] N. C. Beaulieu, A. A. Abu-Dayya, and P. J. McLane, "Comparison of methods of computing lognormal sum distributions and outages for digital wireless applications," in Proc. 1994 IEEE Int. Conf. Communications, New Orleans, LA, May 1994, pp. 1270-1275.
Venugopal V. Veeravalli (S'84-M'92-SM'98) received the B.Tech. degree in 1985 from the Indian Institute of Technology, Bombay (Silver Medal Honors), India, the M.S. degree in 1987 from Carnegie-Mellon University, Pittsburgh, PA, and the Ph.D. degree in 1992 from the University of Illinois at UrbanaChampaign, all in electrical engineering.

He joined Cornell University, Ithaca, NY, in 1996, where he is currently an Assistant Professor of Electrical Engineering and a member of the graduate fields of Statistics and Applied Mathematics. He has been a Post-Doctoral Fellow at Harvard University, Cambridge, MA, an Assistant Professor at the City College of New York, and a Visiting Assistant Professor at Rice University, Houston, TX. He teaches and conducts research in mobile and wireless communication systems and related topics in communication theory. His current topics of research include: channel modeling, bandwidth-efficient modulation and coding, capacity analysis, and dynamic radio resource management for wireless communication systems; decentralized dynamic decision making; and sequential multihypothesis testing.

Dr. Veeravalli is a corecipient of the 1996 IEEE Browder J. Thompson Award. In 1998, he received a CAREER Award from the National Science Foundation and the PECASE (Presidential Early Career Award for Scientists and Engineers) from the White House.

Andrew Sendonaris (S'93-M'99) received the B.S. and M.S. degrees (summa cum laude) in 1993 and 1995, respectively, and the Ph.D. degree in 1999, all in electrical engineering, from Rice University, Houston, TX.

While at Rice University, he was a Research Assistant with the Center for Multimedia Communications where his research interests included multipleaccess communications over multipath fading channels, code division multiple access systems, the capacity of and resource allocation for cellular mobile radio networks, and digital transmission over twisted-pair copper loops. He is currently a Senior Engineer at Qualcomm, Inc., Santa Clara, CA, working on third-generation wireless telephony standards. He was featured in the article "Engineers of the Future" in the twenty-fifth anniversary issue of the Electrical Engineering Times in October 1997. The basic concept from his work with Dr. Veeravalli on joint signaling strategies for increasing the capacity of twisted-pair copper cables is being used in the standardization of HDSL2 second-generation high-bit-rate digital subscriber lines. He has five patents pending, all related to cellular system analysis and design, which were filed during a summer internship at Nortel Networks. 